\title{
NECESSARY CONDITIONS FOR THE CONVERGENCE OF CARDINAL HERMITE SPLINES AS THEIR DEGREE TENDS TO INFINITY \\ BY
}

T. N. T. GOODMAN

\begin{abstract}
Let $\delta_{n,}$ denote the class of cardinal Hermite splines of degree $n$ having knots of multiplicity $s$ at the integers. In this paper we show that if $f_{n} \rightarrow f$ uniformly on $\mathbf{R}$, where $f_{n} \in \delta_{i_{n},} i_{n} \rightarrow \infty$ as $n \rightarrow \infty$, and $f$ is bounded, then $f$ is the restriction to $R$ of an entire function of exponential type $<s$. In proving this result, we need to derive some extremal properties of certain splines $\mathcal{E}_{n, s} \in \mathcal{S}_{n, s}$, in particular that $\left\|\mathcal{E}_{n, s}\right\|_{\infty}$ minimises $\|S\|_{\infty}$ over $S \in$ $\delta_{n, s}$ with $\left\|S^{(n)}\right\|_{\infty}=\left\|\delta_{n, s}^{(n)}\right\|_{\infty}$
\end{abstract}

1. Introduction. For $n=1,2, \ldots$ and $1 \leqslant s \leqslant n$, let

$\mathscr{F}_{n, s}=\left\{f \in C^{n-s}(\mathbf{R}): f \mid(\nu, \nu+1) \in C^{n-1}[(\nu, \nu+1)]\right.$ and

$$
f^{(n-1)} \text { absolutely continuous on }(\nu, \nu+1), \forall \nu \in \mathbf{Z} \text {. }
$$

We let $\mathcal{S}_{n, s}$ denote the set of all cardinal spline functions of degree $n$ in $\mathscr{F}_{\text {n,s }}$, i.e.,

$$
\mathcal{S}_{n, s}=\left\{S \in C^{n-s}(\mathbf{R}): S \mid(\nu, \nu+1) \in \pi_{n}, \forall \nu \in \mathbf{Z}\right\},
$$

where $\pi_{n}$ denotes the set of all polynomials of degree at most $n$.

Throughout this paper, $\|f\|$ will denote ess $\sup _{x \in \mathbf{R}}|f(x)|$.

In [6] Lipow and Schoenberg have shown that for odd $n, 1<s<\frac{1}{2}(n+1)$, and any function $f$ with $f^{(v)}$ of power growth on $\mathbf{R}, \nu=0,1, \ldots, s-1$, there is a unique $S_{n, s} \in \mathcal{S}_{n, s}$ of power growth such that $S_{n, s}^{(v)}$ interpolates $f^{(v)}$ at the integers. In [8] Marsden and Riemenschneider have shown that if $f$ is the Fourier-Stieltjes transform of a measure on $(-s \pi, s \pi)$, then $S_{n, s}^{(v)} \rightarrow f^{(v)}$ uniformly on $\mathbf{R}$ as $n \rightarrow \infty, \nu=0,1, \ldots, s-1$. The case $s=1$ had previously been proved by Schoenberg [10] who established in [11] the partial converse that if $f$ is bounded on $\mathbf{R}$ and $S_{n, 1} \rightarrow f$ uniformly on $\mathbf{R}$ as $n \rightarrow \infty$, then $f$ is the restriction to $\mathbf{R}$ of an entire function of exponential type $<\pi$.

In $\$ 4$ of this paper we generalise Schoenberg's result by showing, in particular, that for any $s=1,2, \ldots$, if $f$ is bounded on $\mathbf{R}$ and $S_{n, s} \rightarrow f$ uniformly on $\mathbf{R}$ as $n \rightarrow \infty$, then $f$ is the restriction to $\mathbf{R}$ of an entire function

Received by the editors August 18, 1978.

AMS (MOS) subject classifications (1970). Primary 41A15; Secondary 41A05.

Key words and phrases. Cardinal spline interpolation, cardinal Hermite splines, Euler splines, Chebyshev polynomials. 
of exponential type $\leqslant s \pi$. To establish this result we need some extremal properties of certain splines $\mathcal{E}_{n, s} \in \mathcal{S}_{n, s}$ which may be regarded as generalisations of the Euler splines employed in [11]. For odd $s$ these were defined by Cavaretta in [1]. In $\$ 2$ we define $\varepsilon_{n, s}$ for even $s$ and show that for all $s$, $f \in \mathscr{F}_{n, s},\|f\|<1=\left\|\mathcal{E}_{n, s}\right\|$ and $\left\|f^{(n)}\right\|<\left\|\mathcal{E}_{n, s}^{(n)}\right\|$ implies

$$
\left|f^{(k)}(\nu+)\right|<\left|\mathcal{E}_{n, s}^{(k)}(\nu+)\right|, \quad \forall \nu \in \mathbf{Z} \text { and } k=n-s, \ldots, n-1 .
$$

In [1] Cavaretta shows that for odd $s, S=\mathcal{E}_{n, s}$ minimises $\|S\|$ over all $S \in \mathcal{S}_{n, s}$ with

$$
S^{(n)} \mid(\nu, \nu+1)=(-1)^{\nu}\left\|\delta_{n, s}^{(n)}\right\|, \quad \forall \nu \in \mathbf{Z} .
$$

In $\$ 3$ we show that for all $s, S=\varepsilon_{n, s}$ actually minimises $\|S\|$ over all $S \in \mathcal{S}_{n, s}$ with $\left\|S^{(n)}\right\|=\left\|\mathcal{E}_{n, s}^{(n)}\right\|$.

2. The Euler-Chebyshev splines. In [1] Cavaretta shows there are functions $\mathcal{E}_{n, s}$ in $\delta_{n, s}$ for $n=1,2, \ldots$ and odd $s<n$, characterised by the following properties:

$$
\mathcal{E}_{n, s}(x+1)=(-1)^{s} \mathcal{E}_{n, s}(x), \quad \forall x \in \mathbf{R},
$$

$\mathcal{E}_{n, s}(x)$ equioscillates between -1 and 1 at points

$$
0<\beta_{1}<\cdots<\beta_{s}<1,
$$

$\varepsilon_{n, s}$ is even or odd about $x=\frac{1}{2}$ as $n$ is even or odd,

$$
\mathcal{E}_{n, s}^{(n)}(x)>0 \text { on }(0,1) \text {. }
$$

We now construct functions $\mathcal{E}_{n, s}$ in $\delta_{n, s}$ for $n=1,2, \ldots$ and even $s<n$ which are also characterised by properties (2.1)-(2.4).

We shall need the following lemma. Its proof is almost identical to that of Proposition 1 in [1] and so will be omitted.

LEMMA 1. Let $\left\{f_{1}(x), \ldots, f_{k}(x)\right\}$ be a Chebyshev system in $[a, b]$ and define

$$
g_{i}(x)=(x-a)(x-b) f_{i}(x), \quad i=1, \ldots, k .
$$

Let $F(x)$ be a continuous function on $[a, b]$ which vanishes at $a$ and $b$. Then there exists a unique linear combination $\sum_{i=1}^{k} a_{i} g_{i}(x)$ of best approximation in the uniform norm to $F(x)$. This best approximation is uniquely characterised by $a(k+1)$-point equioscillation property, i.e. there exist $k+1$ points $a<x_{1}$ $<\cdots<x_{k+1}<b$ where the error function assumes the value of its norm with alternating signs.

We first consider the case of odd $n$. For any $p, q, 1<q<p$, we define

$$
\begin{aligned}
V_{2 p+1,2 q}=\left\{f \in \pi_{2 p+1} \mid\left[0, \frac{1}{2}\right]: f^{(2 i)}(0)=0, i\right. & =0, \ldots, p-q, \\
f^{(2 j)}\left(\frac{1}{2}\right) & =0, j=0, \ldots, p\} .
\end{aligned}
$$

It follows from the theory of Jerome and Schumaker [3] and Lorentz [7] 
that $\operatorname{dim} V_{2 p+1,2 q}=q$ and any $f$ in $V_{2 p+1,2 q}$ has at most $q+1$ zeros in $\left[0, \frac{1}{2}\right]$. Thus if $x\left(x-\frac{1}{2}\right) f_{i}(x), i=1, \ldots, q$, form a basis for $V_{2 p+1,2 q}$, then $\left\{f_{1}(x), \ldots, f_{q}(x)\right\}$ form a Chebyshev system on $\left[0, \frac{1}{2}\right]$.

Now take any odd $n$ and even $s, 4<s<n$, and take any $f$ in $V_{n, s}$ with $f^{(n)}>0$. Let $F$ denote the best approximation to $f$ in the uniform norm in $V_{n-2, s-2}$. Then by Lemma $1, f-F$ equioscillates at points $0<\beta_{1}<\cdots<$ $\beta_{s / 2}<\frac{1}{2}$. Let $G=(f-F) /\|f-F\|$ and define $\mathcal{E}_{n, s}$ in $\mathcal{S}_{n, s}$ by

$$
\begin{aligned}
\mathcal{E}_{n, s}(x) & = \begin{cases}G(x), & 0<x<\frac{1}{2}, \\
(-1)^{n} G(1-x), & \frac{1}{2}<x<1,\end{cases} \\
\mathcal{E}_{n, s}(x+1) & =\mathcal{E}_{n, s}(x), \quad \forall x \in \mathbf{R} .
\end{aligned}
$$

For $s=2$, let $G$ be the element of $V_{n, 2}$ with $\|G\|=1$ and $G^{(n)}>0$, and again define $\varepsilon_{n, s}$ by (2.5). Since $G(0)=G\left(\frac{1}{2}\right)=0, \exists \beta_{1} \in\left(0, \frac{1}{2}\right)$ with $\left|G\left(\beta_{1}\right)\right|$ $=1$, and so $\mathcal{E}_{n, 2}$ equioscillates at $\beta_{1}$ and $\beta_{2}=1-\beta_{1}$. Thus for all even $s, \mathcal{E}_{n, s}$ is characterised by properties (2.1) to (2.4).

Next consider even $n$. For any $p, q, 0<q<p$, define

$$
\begin{aligned}
V_{2 p, 2 q}=\left\{f \in \pi_{2 p} \mid\left[0, \frac{1}{2}\right]: f^{(2 i+1)}(0)\right. & =0, \quad i=0, \ldots, p-q-1, \\
f^{(2 j+1)}\left(\frac{1}{2}\right) & =0, j=0, \ldots, p-1\} .
\end{aligned}
$$

Then $\operatorname{dim} V_{2 p, 2 q}=q+1$ and any $f$ in $V_{2 p, 2 q}$ has at most $q$ zeros in $\left[0, \frac{1}{2}\right]$. Thus any basis for $V_{2 p, 2 q}$ forms a Chebyshev system.

Now take even $n$ and even $s, 2<s<n$, and take any $f$ in $V_{n, s}$ with $f^{(n)}>0$. Let $F$ denote the best approximation to $f$ in the uniform norm in $V_{n-2, s-2}$. Then $f-F$ equioscillates at points $0 \leqslant \beta_{1}<\cdots<\beta_{s / 2+1}<\frac{1}{2}$. Now $f^{\prime}-$ $F^{\prime}$ is in $V_{n-1, s}$ and so has at most $\frac{1}{2} s-1$ zeros in $\left(0, \frac{1}{2}\right)$. Thus $\beta_{1}=0$ and $\beta_{s / 2+1}=\frac{1}{2}$. Let $G=(f-F) /\|f-F\|$ and define $\mathcal{E}_{n, s}$ in $\mathcal{\delta}_{n, s}$ by (2.5). Then again $\mathcal{E}_{n, s}$ is characterised by properties (2.1)-(2.4).

We note that, for $m=1,2, \ldots$,

$$
\begin{aligned}
& \mathcal{E}_{2 m-1,1}(x)=(-1)^{m} \mathcal{E}_{2 m-1}(x), \\
& \mathcal{E}_{2 m, 1}(x)=(-1)^{m} \mathcal{E}_{2 m}\left(x-\frac{1}{2}\right),
\end{aligned}
$$

where $\mathcal{E}_{n}$ denotes the Euler spline of degree $n$, see [11].

We also note that, for $n=1,2, \ldots$,

$$
\mathcal{E}_{n, n}(x)=T_{n}(2 x-1), \quad \forall x \in[0,1],
$$

where $T_{n}$ denotes the Chebyshev polynomial of degree $n$.

It therefore seems appropriate to refer to $\delta_{n, s}$ as Euler-Chebyshev splines, or ET-splines, following the similar terminology introduced by Cavaretta in [1]. They satisfy the following extremal property which is related to a theorem of Kolmogorov (see [2]). 
THEOREM 1. Suppose $f$ in $\mathscr{F}_{n, s}$ satisfies

$$
\|f\|<1 \text { and }\left\|f^{(n)}\right\|<\left\|\mathcal{E}_{n, s}^{(n)}\right\|,
$$

then

$$
\left|f^{(k)}(\nu+)\right|<\left|\mathcal{E}_{n, s}^{(k)}(\nu+)\right|, \quad \forall \nu \in \mathbf{Z}, \quad k=n-s, \ldots, n-1 .
$$

Proof. We use an elementary and powerful technique introduced by Cavaretta [2].

Without loss of generality we may take $\nu=0$. Suppose $f$ in $\mathscr{F}_{n, s}$ satisfies (2.7) and is periodic of period an even integer $K$. We shall assume $\left|f^{(k)}(0+)\right|$ $>\left|\mathcal{E}_{n, s}^{(k)}(0+)\right|$ for some $k, n-s<k<n-1$, and reach a contradiction. Choose $\lambda$ so that $\lambda f^{(k)}(0+)=\mathcal{E}_{n, s}^{(k)}(0+)$ and let $g=\mathcal{E}_{n, s}-\lambda f$, noting that $g$ is also periodic of period $K$.

Since $\|\lambda f\|<\left\|\mathcal{E}_{n, s}\right\|$ and because of the equioscillation of $\mathcal{E}_{n, s}, g$ has at least $K s$ distinct zeros per period. Thus, by repeated application of Rolle's theorem, $g^{(n-s)}$ has at least $K s$ distinct zeros per period. If $k=n-s$, then $g^{(n-s)}(0)=0$ and so $g^{(n-s+1)}$ has at least $K(s-1)+1$ zeros per period which are not at integers. If $k>n-s$, then $g^{(n-s+1)}$ has at least $K(s-1)$ zeros per period which are not at integers, and so $g^{(k)}$ has at least $K(n-k)$ zeros per period which are not at integers. But $g^{(k)}(0+)=0$ and so $g^{(k+1)}$ has at least $K(n-k-1)+1$ changes of sign per period which are not at integers. Thus for all $k, g^{(n)}$ has at least one change of sign per period which is not at an integer. But this contradicts $\left|\lambda f^{(n)}(x)\right|<\mathcal{E}_{n, s}^{(n)}(x) \mid$ in every interval $(\nu, \nu+1)$, $\nu \in \mathbf{Z}$.

We may extend to nonperiodic $f$ in precisely the same manner as in [2].

3. An extremal property of ET-splines. For $n=1,2, \ldots, 1<s<n$, and numbers $\alpha_{1}, \ldots, \alpha_{s}, \lambda$, we define

$$
\begin{aligned}
& \Pi_{n}\left(\alpha_{1}, \ldots, \alpha_{s} ; \lambda\right)
\end{aligned}
$$

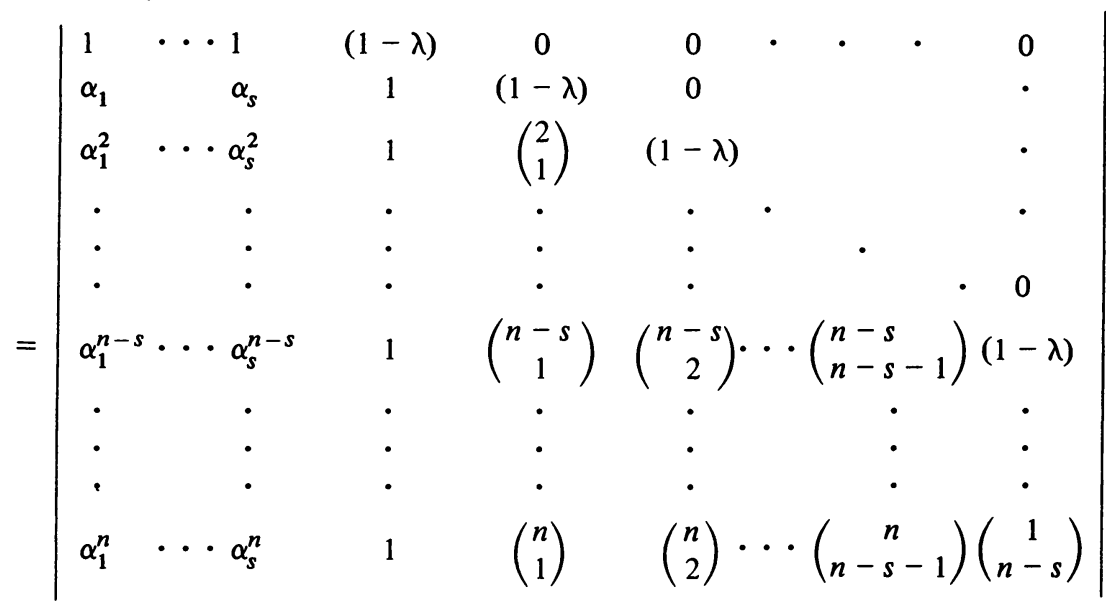


This determinant has the following properties, which follow from the work of Micchelli [9] or by using the method of Lee and Sharma [5].

For fixed $0<\alpha_{1}<\alpha_{2}<\ldots<\alpha_{s}<1, \Pi_{n}(\lambda) \equiv \Pi_{n}\left(\alpha_{1}, \ldots, \alpha_{s} ; \lambda\right)$ is a polynomial in $\lambda$ with real distinct roots of sign $(-1)^{s}$. If $\alpha_{1}>0, \Pi_{n}(\lambda)=$ $a \lambda^{n-s+1}+\ldots$, where sign $a=(-1)^{(s+1)(n+s+1)}$. If $\alpha_{1}=0, \Pi_{n}(\lambda)=a \lambda^{n-s}$ $+\ldots$, where sign $a=(-1)^{(s+1)(n+s)}$. If the nonzero $\alpha_{i}, i=1, \ldots, s$, are symmetric about $\frac{1}{2}$, then $\Pi_{n}(\lambda)$ is reciprocal.

Now fix $0<\alpha_{1}<\alpha_{2}<\cdots<\alpha_{s}<1$ and take $r, 1<r<s$. For $x \in[0,1]$ we define

$$
\begin{aligned}
\Pi(x, \lambda) & =\Pi_{n}\left(\alpha_{1}, \ldots, \alpha_{r-1}, x, \alpha_{r+1}, \ldots \alpha_{s} ; \lambda\right) \\
& =p_{0}(x) \lambda^{n-s+1}+p_{1}(x) \lambda^{n-s}+\cdots+p_{n-s+1}(x) .
\end{aligned}
$$

Then it is easy to show that

$$
\frac{\partial^{j}}{\partial x^{j}} \Pi(1, \lambda)=\lambda \frac{\partial^{j}}{\partial x^{j}} \Pi(0, \lambda), \quad j=0, \ldots, n-s,
$$

and

$$
\Pi\left(\alpha_{i}, \lambda\right)=0, \quad i \neq r .
$$

We now define the ' $B$-spline'

$$
B_{r}(x)=\left\{\begin{array}{l}
p_{\nu}(x-\nu), \quad x \in[\nu, \nu+1), \quad \nu=0, \ldots, n-s+1, \\
0, \quad x<0 \text { and } x>n-s+2 .
\end{array}\right.
$$

From (3.1) we see that $B_{r} \in \mathcal{S}_{n, s}$ and from (3.2) we have $B_{r}\left(\alpha_{i}+\nu\right)=0$ for all $\nu \in \mathbf{Z}$ and $i \neq r$. Also

$$
\sum_{\nu=-\infty}^{\infty} B_{r}(x+\nu) t^{\nu}=t^{n-s+1} \Pi\left(x, t^{-1}\right), \quad x \in[0,1) .
$$

Now assume

$$
\Pi_{n}\left(\alpha_{1}, \ldots, \alpha_{s} ;(-1)^{s}\right) \neq 0
$$

Then following the method of Schoenberg [11], we may write

$$
\left\{\sum_{\nu}^{\infty} B_{r}\left(\nu+\alpha_{r}\right) t^{\nu}\right\}^{-1}=\sum_{\nu=-\infty}^{\infty} \omega_{\nu} t^{\nu}
$$

where the series is convergent on some annulus about $|t|=1$ and $\left|\omega_{p}\right|=$ $O\left(\beta^{\nu}\right)$ as $\nu \rightarrow \pm \infty$ for some $0<\beta<1$.

We now define the 'fundamental spline'

$$
L_{r}(x)=\sum_{\nu=-\infty}^{\infty} \omega_{\nu} B_{r}(x-\nu)
$$

Then

$$
\begin{aligned}
L_{r}\left(k+\alpha_{r}\right) & =\sum_{\nu=-\infty}^{\infty} \omega_{\nu} B_{r}\left(k+\alpha_{r}-\nu\right) \\
& =\delta_{k 0}, \quad \forall k \in \mathbf{Z}, \quad \text { by (3.5). }
\end{aligned}
$$


It follows from the theory of [9] that if $S \in \mathcal{S}_{n, s}$ is of power growth, then

$$
S(x)=\sum_{r=1}^{s} \sum_{k=-\infty}^{\infty} S\left(k+\alpha_{r}\right) L_{r}(x-k) \text {. }
$$

Now take $x$ in $(0,1)$. Then

$$
\frac{\partial^{n}}{\partial x^{n}} \Pi(x, \lambda)=(-1)^{n+r+1} n ! \Pi_{n-1}\left(\alpha_{1}, \ldots, \alpha_{r-1}, \alpha_{r+1}, \ldots \alpha_{s} ; \lambda\right) .
$$

So, by (3.3),

$$
\begin{aligned}
\sum_{\nu=-\infty}^{\infty} B_{r}^{(n)} & (\nu+x) t^{\nu} \\
& =(-1)^{n+r+1} n ! t^{n-s+1} \Pi_{n-1}\left(\alpha_{1}, \ldots, \alpha_{r-1}, \alpha_{r+1}, \ldots, \alpha_{s} ; t^{-1}\right)
\end{aligned}
$$

Now

$$
L_{r}^{(n)}(k+x)=\sum_{\nu=-\infty}^{\infty} \omega_{\nu} B_{r}^{(n)}(k+x-\nu)
$$

and so

$$
\sum_{k=-\infty}^{\infty} L_{r}^{(n)}(k+x) t^{k}=\left(\sum_{i=-\infty}^{\infty} \omega_{i} t^{i}\right)\left(\sum_{j=-\infty}^{\infty} B_{r}^{(n)}(j+x) t^{j}\right) .
$$

So by (3.7), (3.5) and (3.3),

$$
\begin{aligned}
\sum_{k=-\infty}^{\infty} L_{r}^{(n)}(k+x) t^{k} & \\
& =\frac{(-1)^{n+r+1} n ! \Pi_{n-1}\left(\alpha_{1}, \ldots, \alpha_{r-1}, \alpha_{r+1}, \ldots, \alpha_{s} ; t^{-1}\right)}{\Pi_{n}\left(\alpha_{1}, \ldots, \alpha_{s} ; t^{-1}\right)} .
\end{aligned}
$$

Then from (3.8) and the properties of $\Pi_{n}(\lambda)$, we have the following result.

$$
\begin{aligned}
\sum_{k=-\infty}^{\infty} L_{r}^{(n)}(k+x) t^{k} & =\frac{(-1)^{r+s} K \Pi_{i=1}^{n-s+1}\left(1+\mu_{i} t\right)}{\Pi_{j=1}^{n-s+1}\left(1-\lambda_{j} t\right)} \quad \text { if } \alpha_{1}>0, \\
& =\frac{(-1)^{r+s+1} K \Pi_{i=1}^{n-s}\left(1+\mu_{i} t\right)}{\Pi_{j=1}^{n-s}\left(1-\lambda_{j} t\right)} \quad \text { if } \alpha_{1}=0, \quad r>1, \\
& =\frac{K \Pi_{i-1}^{n-s+1}\left(1+\mu_{i} t\right)}{t \Pi_{j=1}^{n-s}\left(1-\lambda_{j} t\right)} \text { if } \alpha_{1}=0, \quad r=1,
\end{aligned}
$$

where $K, \mu_{i}, \lambda_{j}$ are constants (depending on $r, n, \alpha_{1}, \ldots, \alpha_{s}$ ) with $K>0$ and $\operatorname{sign} \mu_{i}=\operatorname{sign} \lambda_{j}=(-1)^{s}, \forall i, j$.

We therefore have (see $[4$, p. 395]),

$$
\operatorname{sign} L_{r}^{(n)}(k+x)= \begin{cases}(-1)^{q+r+k} & s \text { odd, } \\ (-1)^{q+r+1}, & s \text { even, }\end{cases}
$$


where

$$
q= \begin{cases}1, & \text { if } \alpha_{1}>0 \\ 0, & \text { if } \alpha_{1}=0\end{cases}
$$

We are now in a position to prove our result.

THEOREM 2. If $S \in \mathcal{S}_{n, s}$ satisfies $\|S\|<1$, then $\left\|S^{(n)}\right\|<\left\|\mathcal{\delta}_{n, s}^{(n)}\right\|$.

Proof. Take $\beta_{1}, \ldots, \beta_{s}$ as in (2.2). By (2.3) we know the nonzero $\beta_{i}$, $i=1, \ldots, s$, are symmetric about $\frac{1}{2}$ and so $\Pi_{n}\left(\beta_{1}, \ldots, \beta_{s} ; \lambda\right)$ is a reciprocal polynomial in $\lambda$. If $n$ and $s$ are both even or both odd, then $\beta_{1}=0$. Otherwise $\beta_{1}>0$. Thus in all cases, $\Pi_{n}\left(\beta_{1}, \ldots, \beta_{s} ; \lambda\right)$ is a polynomial in $\lambda$ of even degree and so

$$
\Pi_{n}\left(\beta_{1}, \ldots, \beta_{s} ;(-1)^{s}\right) \neq 0 .
$$

Since (3.4) is satisfied, we may define the 'fundamental spline' $L_{r}$ for $r=1, \ldots, s$. Then for any $S \in \mathcal{S}_{n, s}$ satisfying $\|S\|<1$, we have from (3.6),

$$
\begin{aligned}
\left|S^{(n)}(x)\right| & =\left|\sum_{r=1}^{s} \sum_{k=-\infty}^{\infty} S\left(k+\beta_{r}\right) L_{r}^{(n)}(x-k)\right| \\
& <\sum_{r=1}^{s} \sum_{k=-\infty}^{\infty}\left|L_{r}^{(n)}(x-k)\right|, \quad \forall x \in \mathbf{R} .
\end{aligned}
$$

But it follows from (3.9) and (2.2) that equality is attained in (3.10) for $S=\varepsilon_{n, s}$.

For $s=1$ this result was proved by Schoenberg [11], and for $s=n$ the result follows immediately from the properties of Chebyshev polynomials.

It is clear from the proof of Theorem 2 that the condition $\|S\|<1$ in the statement of the theorem can be replaced by the weaker condition

$$
\left|S\left(k+\beta_{i}\right)\right|<1, \quad \forall k \in \mathbf{Z}, \quad i=1, \ldots, s .
$$

4. Limits of cardinal splines. We need a further property of ET-splines.

LEMMA 2. For $s=1,2, \ldots$, there are constants $K_{s}$ such that $\left\|\mathcal{E}_{n, s}^{(v)}\right\|<$ $K_{s}(s \pi)^{n}$ for all $n>s$ and $\nu=0, \ldots, n$.

Proof. First suppose $s$ is odd, $s=2 t-1$. It follows from the work of [1] that for any $n>s$,

$$
\varepsilon_{n, s}=\varepsilon_{n, 1}+\mu_{1} \varepsilon_{n-2,1}+\cdots+\mu_{t-1} \varepsilon_{n-2 t+2,1},
$$

where $\mu_{1}, \ldots, \mu_{t-1}$ are chosen to minimise $\left\|\mathcal{E}_{n, s}\right\|$.

We first consider odd $n>s$. Then it follows from (4.1) and (2.6) that we may write

$$
\mathcal{E}_{n, s}=(-1)^{(n+1) / 2} \phi_{n} /\left\|\phi_{n}\right\|
$$


where

$$
\begin{aligned}
\phi_{n}(x)= & \sum_{r=1}^{\infty} \frac{\cos (2 r-1) \pi x}{(2 r-1)^{n+1}}+\lambda_{1}^{(n)} \sum_{r=1}^{\infty} \frac{\cos (2 r-1) \pi x}{(2 r-1)^{n-1}} \\
& +\cdots+\lambda_{t-1}^{(n)} \sum_{r=1}^{\infty} \frac{\cos (2 r-1) \pi x}{(2 r-1)^{n-2 t+3}} \\
= & \sum_{r=1}^{\infty} \frac{\cos (2 r-1) \pi x}{(2 r-1)^{n+1}}\left\{1+\lambda_{1}^{(n)}(2 r-1)^{2}+\cdots+\lambda_{t-1}^{(n)}(2 r-1)^{2 t-2}\right\},
\end{aligned}
$$

and $\lambda_{1}^{(n)}, \ldots, \lambda_{t-1}^{(n)}$ are chosen to minimise $\left\|\phi_{n}\right\|$.

Let $\lambda_{1}, \ldots, \lambda_{t-1}$ be the unique solution of the equations

$$
1+(2 r-1)^{2} \lambda_{1}+\cdots+(2 r-1)^{2 t-2} \lambda_{t-1}=0, \quad r=1, \ldots, t-1 .
$$

Let

$$
\psi_{n}(x)=\sum_{r=t}^{\infty} \frac{\cos (2 r-1) \pi x}{(2 r-1)^{n+1}}\left\{1+\lambda_{1}(2 r-1)^{2}+\cdots+\lambda_{t-1}(2 r-1)^{2 t-2}\right\} \text {. }
$$

Then $\left\|(2 t-3)^{n+1} \psi_{n}\right\| \rightarrow 0$ as $n \rightarrow \infty$. Since $\left\|\phi_{n}\right\|<\left\|\psi_{n}\right\|,\left\|(2 t-3)^{n+1} \phi_{n}\right\| \rightarrow 0$ as $n \rightarrow \infty$ and so for $r=1, \ldots, t-1$,

$$
\left(\frac{2 t-3}{2 r-1}\right)^{n+1}\left\{1+\lambda_{1}^{(n)}(2 r-1)^{2}+\cdots+\lambda_{t-1}^{(n)}(2 r-1)^{2 t-2}\right\} \rightarrow 0 \text { as } n \rightarrow \infty .
$$

So $\lambda_{i}^{(n)} \rightarrow \lambda_{i}$ as $n \rightarrow \infty, i=1, \ldots, t-1$. Thus

$$
(2 t-1)^{n+1} \phi_{n}(x)=f_{n}(x)+a_{n} \cos (2 t-1) \pi x+O\left(\left[\frac{2 t-1}{2 t+1}\right]^{n}\right)
$$

where $f_{n}(x)$ is of the form $\Sigma_{r=1}^{t-1} b_{r} \cos (2 r-1) \pi x$ and

$$
a_{n} \rightarrow a=1+(2 t-1)^{2} \lambda_{1}+\cdots+(2 t-1)^{2 t-2} \lambda_{t-1} \neq 0 \text { as } n \rightarrow \infty \text {. }
$$

Now for each $n$, there is an integer $j, 1<j<2 t-1$, such that

$$
f_{n}\left(\frac{j}{2 t-1}\right) a_{n} \cos j \pi>0
$$

and so

$$
(2 t-1)^{n+1}\left|\phi_{n}\left(\frac{j}{2 t-1}\right)\right|>\left|a_{n}\right|+O\left(\left[\frac{2 t-1}{2 t+1}\right]^{n}\right) .
$$

So $\exists \delta>0$ such that

$$
s^{n+1}\left\|\phi_{n}\right\|>\delta, \quad \forall n>s .
$$

Writing

$$
g_{n}(x)=\sum_{r=1}^{\infty} \frac{\cos (2 r-1) \pi x}{(2 r-1)^{n+1}}
$$


we have

$$
\left\|g_{n}^{(v)}\right\|<\pi^{\nu}\left(1+\frac{1}{3^{2}}+\frac{1}{5^{2}}+\cdots\right)<2 \pi^{\nu}
$$

for $n=1,2, \ldots$ and $\nu<n$. Also $\left\|g_{n}^{(n)}\right\|=2\left\|g_{n}^{(n-1)}\right\|<4 \pi^{n-1}$. So

$$
\left\|\phi_{n}^{(v)}\right\|<4 \pi^{n-1}\left\{1+\left|\lambda_{1}^{(n)}\right|+\cdots+\left|\lambda_{t-1}^{(n)}\right|\right\}, \quad \nu<n,
$$

and so there is a constant $K$ such that

$$
\left\|\phi_{n}^{(\nu)}\right\|<K \pi^{n} \text { for all } n>s \text { and } \nu<n .
$$

Thus

$$
\left\|\mathcal{E}_{n, s}^{(v)}\right\|=\left\|\phi_{n}^{(\nu)}\right\| /\left\|\phi_{n}\right\|<\frac{K s}{\delta}(s \pi)^{n}, \quad \forall n>s, \quad \nu<n,
$$

by (4.2) and (4.3).

The result for even $n$ follows similarly.

Next suppose $s$ is even, $s=2 t$. We first note that

$$
\mathcal{\delta}_{n, 2}^{(n-1)}(x) /\left\|\mathcal{G}_{n, 2}^{(n)}\right\|=x-\frac{1}{2}, \quad \forall x \in(0,1) .
$$

So

where

$$
\mathcal{E}_{n, 2}=(-1)^{[n / 2]} h /\|h\|,
$$

$$
h(x)=\left\{\begin{array}{l}
\sum_{k=1}^{\infty} \frac{(-1)^{k+1}}{k^{n}} \cos 2 k \pi\left(x-\frac{1}{2}\right)+\sum_{k=1}^{\infty} \frac{1}{(2 k)^{n}} \quad \text { if } n \text { even } \\
\sum_{k=1}^{\infty} \frac{(-1)^{k+1}}{k^{n}} \sin 2 k \pi\left(x-\frac{1}{2}\right) \text { if } n \text { odd. }
\end{array}\right.
$$

It follows that for even $n$,

$$
\varepsilon_{n, s}=(-1)^{n / 2} \phi_{n} /\left\|\phi_{n}\right\|,
$$

where

$$
\begin{aligned}
\phi_{n}(x)= & \mu+\sum_{k=1}^{\infty} \frac{(-1)^{k+1}}{k^{n}} \cos 2 k \pi\left(x-\frac{1}{2}\right)+\lambda_{1}^{(n)} \sum_{k=1}^{\infty} \frac{(-1)^{k+1}}{k^{n-2}} \cos 2 k \pi\left(x-\frac{1}{2}\right) \\
& +\cdots+\lambda_{t-1}^{(n)} \sum_{k=1}^{\infty} \frac{(-1)^{k+1}}{k^{n-2 t+2}} \cos 2 k \pi\left(x-\frac{1}{2}\right),
\end{aligned}
$$

and $\mu, \lambda_{1}^{(n)}, \ldots, \lambda_{l-1}^{(n)}$ are chosen to minimise $\left\|\phi_{n}\right\|$.

For odd $n$,

$$
\mathcal{E}_{n, s}=(-1)^{(n-1) / 2} \phi_{n} /\left\|\phi_{n}\right\|
$$


where

$$
\begin{aligned}
\phi_{n}(x)= & \sum_{k=1}^{\infty} \frac{(-1)^{k+1}}{k^{n}} \sin 2 k \pi\left(x-\frac{1}{2}\right)+\lambda_{1}^{(n)} \sum_{k=1}^{\infty} \frac{(-1)^{k+1}}{k^{n}} \sin 2 k \pi\left(x-\frac{1}{2}\right) \\
& +\cdots+\lambda_{t-1}^{(n)} \sum_{k=1}^{\infty} \frac{(-1)^{k+1}}{k^{n-2 t+2}} \sin 2 k \pi\left(x-\frac{1}{2}\right),
\end{aligned}
$$

and $\lambda_{1}^{(n)}, \ldots, \lambda_{l-1}^{(n)}$ are chosen to minimise $\left\|\phi_{n}\right\|$.

The result now follows by the same method as for odd $s$.

We now apply Lemma 2 and Theorems 1 and 2 in proving the following:

LEMMA 3. For $s=1,2, \ldots$, there are constants $L_{s}$ such that if $S$ in $\delta_{n, s}$ satisfies $\|S\|<1$, then $\left\|S^{(k)}\right\|<L_{s}(s \pi)^{k}$, for all $n>s$ and $k<n-s$.

Proof. Take $S$ in $\delta_{n, s}$ with $\|S\|<1$. Then by Theorem 2, $\left\|S^{(n)}\right\|<\left\|\delta_{n, s}^{(n)}\right\|$. So by Theorem 1 ,

$$
\left|S^{(k)}(\nu+)\right| \leqslant\left|\mathcal{E}_{n, s}^{(k)}(\nu+)\right|, \quad \forall \nu \in \mathbf{Z}, \quad k=n-s+1, \ldots, n-1 .
$$

So by Lemma 2 ,

$$
\left\|S^{(n)}\right\|<K_{s}(s \pi)^{n}
$$

and

$$
\left|S^{(k)}(\nu+)\right|<K_{s}(s \pi)^{n}, \quad \forall \nu \in \mathbf{Z}, \quad k=n-s+1, \ldots, n-1 .
$$

It follows from (4.4) and (4.5) for $k=n-1$ that $\left\|S^{(n-1)}\right\|<2 K_{s}(s \pi)^{n}$. Proceeding in this manner we deduce that

$$
\left\|S^{(n-s+1)}\right\|<s K_{s}(s \pi)^{n} .
$$

Let $T(x)=S(M x)$, where $M=\left[\frac{1}{2} K_{s} s^{n+1} \pi^{s}\right]^{-1 /(n-s+1)}$. Then

$$
\begin{aligned}
\left|T^{(n-s+1)}(x)\right| & =M^{n-s+1}\left|S^{(n-s+1)}(x)\right| \\
& <\left[\frac{1}{2} K_{s} s^{n+1} \pi^{s}\right]^{-1} s K_{s}(s \pi)^{n} \\
& =2 \pi^{n-s}<\left\|\mathcal{E}_{n-s+1}^{(n-s+1)}\right\| .
\end{aligned}
$$

So by a theorem of Kolmogorov (see [2]), for $k<n-s$,

$$
\left\|T^{(k)}\right\|<\left\|\delta_{n-s+1}^{(k)}\right\|<2 \pi^{k} \quad(\text { see }[11]) .
$$

So

$$
\begin{aligned}
\left\|S^{(k)}\right\| & =M^{-k}\left\|T^{(k)}\right\|<M^{-k} 2 \pi^{k} \quad(\text { by }(4.7)) \\
& =2\left[\frac{1}{2} K_{s}(s \pi)^{s}\right]^{k /(n-s+1)}(s \pi)^{k}<L_{s}(s \pi)^{k}
\end{aligned}
$$

where $L_{s}=\max \left\{2, K_{s}(s \pi)^{s}\right\}$.

By the method of Schoenberg [11], we may deduce from Lemma 3 our final result. 
THEOREM 3. For a given natural number $s$, suppose $f_{n} \in \mathcal{S}_{i_{n}, s}$, where $i_{n} \rightarrow \infty$ as $n \rightarrow \infty$. If $f_{n} \rightarrow f$ uniformly on $\mathbf{R}$ and $f$ is bounded, then $f$ is the restriction to $\mathbf{R}$ of an entire function of exponential type $<s$.

Acknowledgement. The author wishes to thank S. L. Lee for drawing his attention to the problems considered here and for many helpful discussions.

\section{REFERENCES}

1. A. S. Cavaretta, Jr., On cardinal perfect splines of least sup-norm on the real axis, J. Approximation Theory 8 (1973), 285-303.

2. , An elementary proof of Kolmogorov's theorem, Amer. Math. Monthly 82 (1974), 480-486.

3. J. W. Jerome and L. L. Schumaker, On Lg-splines, J. Approximation Theory 2 (1969), 29-49.

4. S. Karlin, Total positivity, vol. 1, Stanford Univ. Press, Stanford, California, 1968.

5. S. L. Lee and A. Sharma, Cardinal lacunary interpolation by 8 -splines. I, The characteristic polynomials, J. Approximation Theory 16 (1976), 85-96.

6. P. R. Lipow and I. J. Schoenberg, Cardinal interpolation and spline functions. III, Cardinal Hermite interpolation, Linear Algebra and Appl. 6 (1973), 273-304.

7. G. G. Lorentz, Zeros of splines and Birkhoff's kernel, Math. Z. 142 (1975), 173-180.

8. M. J. Marsden and S. D. Riemenschneider, Cardinal Hermite spline interpolation. Convergence as the degree tends to infinity, Trans. Amer. Math. Soc. 235 (1973), 221-244.

9. C. A. Micchelli, Oscillation matrices and cardinal spline interpolation, Splines and Approximation Theory, Academic Press, New York, 1976.

10. I. J. Schoenberg, Notes on spline functions. III, On the convergence of the interpolating cardinal splines as their degree tends to infinity, Israel J. Math. 16 (1973), 87-93.

11. Notes on spline functions. IV, A cardinal spline analogue of the theorem of the brothers Markov, Israel J. Math. 16 (1973), 94-102.

School of Mathematical Sciences, University of Science of Malaysia, Penang, Malaysia

Current address: Department of Mathematics, The University, Dundee DD1 4HN, Scotland 